\title{
Warming the world
}

\section{Greenhouse effect: Fourier's concept of planetary energy balance is still relevant today.}

\section{Raym} Mmond T. Pierrehumbert

ean-Baptiste Joseph Fourier is generally credited with the discovery of the greenhouse effect, whereby the presence of an atmosphere acts to increase a planet's surface temperature. Written in 1827, nearly threequarters of a century before science advanced to the point where Arrhenius could quantify the phenomenon, how well does Fourier's concept measure up against our current understanding of the greenhouse effect?

First, it is important to recognize what Fourier did not do in his 1827 essay. He did not say that the operation of the atmosphere is analogous to that of a greenhouse - the French word serre (greenhouse) does not appear anywhere in the essay - so he should not be blamed for the well known shortcomings of the analogy. Neither did he write down any equations describing the greenhouse effect, nor compute any estimate of planetary temperature.

"In the present work, I have set myself another goal, that of calling attention to one of the greatest objects of natural philosophy," Fourier writes, referring to the problem of planetary temperatures. Thus, the main contribution of the article is the introduction of planetary temperature as a proper object of study in physics. Fourier established the framework of energy balance still in use today: a planet obtains energy at a certain rate from various sources, and warms up until it loses heat at the same rate. Fourier correctly deduced that a planet loses heat almost exclusively by infrared radiation ("chaleur obscure" or 'dark heat') and can do so in a vacuum. Infrared had been discovered by Frederick Herschel only 25 years earlier, and the study of its properties occupied much of the attention of nineteenth-century physicists, including Fourier himself - the long gestation culminated in the birth of quantum theory at the dawn of the twentieth century.

Concerning the Earth's heat source, Fourier first made use of his earlier work on heat diffusion to correctly deduce that the internal heat remaining from the formation of the Earth no longer has a significant influence on surface temperature. He recognized that sunlight carries heat, that the atmosphere is essentially transparent to sunlight, that the light is converted to infrared on being absorbed by the surface, and that the atmosphere is relatively opaque to the infrared that

serves to carry the received heat away to space. In consequence, Fourier reasoned, the temperature has to increase (compared with the no-atmosphere case) to allow sufficient infrared radiation to bring the heat budget into balance. Fourier knew that infrared flux increases with temperature, but had no notion of the form of the increase. Another fifty years were to pass before the discovery of the crucial Stefan-Boltzmann fourth-power law.

Recognizing the inadequate state of infrared theory, Fourier turned to an experi-

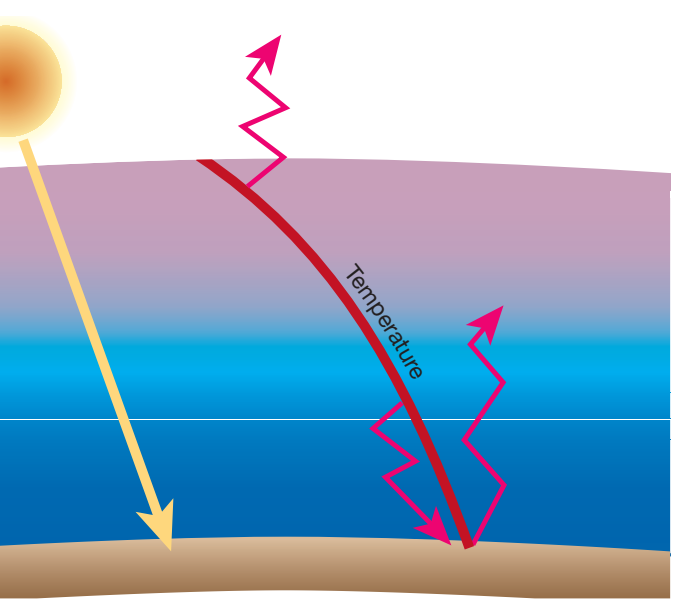

Heat is lost by infrared radiation (pink arrows), but some is absorbed the atmosphere, raising the temperature until output matches input.

black-body radiation law, other phenomena not understood at Fourier's time include the role of convection in causing atmospheric temperature to decrease with height, the importance of this decrease in reducing the mean temperature at which the planet radiates to space, the role of minor atmospheric constitutents (notably carbon dioxide and water vapour) in determining the infrared opacity, quantum theory relating to infrared absorption and emission, the dynamic nature of water vapour and its consequent radiative feedback, and both optical and microphysical properties of clouds. Fourier's essay set the agenda for much of this work. Inadequate understanding of vertical temperature gradient, water vapour and clouds continues to plague our theories of climate.

Just as important as what Fourier got right is what he got spectacularly wrong. Fourier believed that the Earth receives a significant amount of heat directly from interplanetary space, which he supposed to have a temperature comparable to that of the polar winter. The idea is not in itself preposterous, but what is remarkable is that, in coming to this conclusion, Fourier dismissed without cause alternative explanations he knew

ment by the geologist Horace Bénédict de Saussure. The apparatus for de Saussure's experiment consisted of an insulated box lined with black cork, to which sunlight is admitted at the top through one or more sheets of clear glass. He found that on exposure to sunlight, the interior temperature of the box is greatly elevated, as compared with that found when the glass is removed. De Saussure had built his apparatus as a means of measuring the intensity of solar radiation, but Fourier recognized the implications of the results for the problem of planetary temperatures, in that glass - like the atmosphere - is transparent to sunlight but opaque to infrared. In his discussion of the device, Fourier shows a thorough understanding of the extraneous effects at play, and makes quite clear that it is only the part of the interior warming due to infrared effects that is relevant to the Earth.

Fourier got the essence of the greenhouse effect right - the principle of energy balance and the asymmetric effect of the atmosphere on incoming light versus outgoing infrared. The remaining physics took almost two more centuries to sort out, and the job is still not yet done. As well as the Stefan-Boltzman about, and indeed refers to in the same essay: thermal inertia and atmosphere-ocean heat transport, which keep the poles and the night warm without any need to invoke an influx of heat from interplanetary space. Fourier's problem was that he fell in love with an idea, and was thus blinded to things he knew. An object lesson for today?

What will future generations think of our present fumbling attempts to understand climate and predict its future course? I myself am left with a disconcerting feeling that some future Nature essayist may look back and wonder how we managed to ignore so much evidence that the Earth's climate can change more dramatically and catastrophically than our present models predict.

Raymond T. Pierrehumbert is in the Department of the Geophysical Sciences, the University of Chicago, 5734 S. Ellis Avenue, Chicago, Illinois 60637, USA.

\section{FURTHER READING}

Fourier, J.-B. J. Mémoires d l'Académie Royale des Sciences de l'Institute de France VII, 570-604 (1827); a translation of this essay accompanies this article on Nature's website.

Bard, E. C. R. Geosci. 336, 603-638 (2004). 